\title{
Selective hypertrophy of the lobus caudatus as a novel approach enabling extended right hepatectomy in the presence of a non-perfused left lateral liver lobe
}

\author{
Georgi Atanasov • Moritz Schmelzle • Armin Thelen • \\ Georg Wiltberger · Hans-Michael Hau · Felix Krenzien • \\ Tim-Ole Petersen $\cdot$ Michael Moche $\cdot$ Sven Jonas
}

Received: 27 December 2013/Accepted: 23 June 2014/Published online: 12 July 2014

(C) Springer Japan 2014

\begin{abstract}
Introduction Portal vein embolization (PVE) is a wellestablished technique to enhance functional hepatic reserves of segments II and III before curative extended right hepatectomy for tumors of the right liver lobe. However, an adequate hepatopetal flow of the left lateral portal vein branches is required for a sufficient PVEassociated hypertrophy.

Case report Here, we report a 65 -year old patient suffering from a locally advanced intrahepatic cholangiocarcinoma in the right liver lobe and segment IV. A curative extended right hepatectomy after preoperative PVE of liver segments IV-VIII was initially impossible because of partial thrombosis of the left lateral portal vein branches resulting in an ischemic-type atrophy of segments II and III. However, due to a massive hypertrophy of the caudate lobe following PVE of liver segments IV-VIII, subsequent extended right hepatectomy with intraoperative thrombectomy of segments II and III was made possible.

Conclusions To our knowledge this is the first case in which an extended right hepatectomy for a liver malignancy, in the presence of atrophic left lateral section, was
\end{abstract}

G. Atanasov $(\square) \cdot$ M. Schmelzle · A. Thelen · G. Wiltberger ·

H.-M. Hau · F. Krenzien · S. Jonas

Department of Visceral-, Transplantation-, Thoracic- and

Vascular Surgery, University Hospital Leipzig, Liebigstrasse 20,

04103 Leipzig, Germany

e-mail: georgi.atanasov@medizin.uni-leipzig.de

M. Schmelzle $\cdot$ S. Jonas

Translational Centre for Regenerative Medicine, Leipzig

University, Leipzig, Germany

T.-O. Petersen · M. Moche

Department of Diagnostic and Interventional Radiology,

University Hospital Leipzig, Leipzig, Germany made possible by a massive PVE-associated hypertrophy of the caudate lobe.

Keywords Extended right hepatectomy - Portal vein embolization $\cdot$ PVE $\cdot$ Cholangiocarcinoma

\section{Introduction}

Extended right hepatectomy is required to obtain tumorfree surgical margins in up to one-third of oncological liver resections $[1,2]$. However, major hepatectomies are associated with increased morbidity and mortality because of postoperative liver failure (PLF) [3, 4].

Intrahepatic cholangiocarcinoma (IHC) represents the second most common primary liver cancer and its incidence has risen over the past decades in Western countries [5]. Curative resection represents the treatment of choice for IHC, as it is the only chance of long-term survival with 5-year survival rates ranging between 21 and $35 \%$ [6-10]. Results of radio- and conventional chemotherapy are limited with a median survival of 12 months [11-14].

Due to the mass-forming character of this tumor entity and the late onset of symptoms, patients are frequently diagnosed at an advanced tumor stage with the need of an extended right hepatectomy. In case of a predicted too low future liver remnant volume (FLRV) of left lateral liver segments II and III, the safety of major hepatic resection can be significantly improved by preoperative selective portal vein embolization (PVE) of the right liver lobe.

An adequate hepatopetal flow of the left lateral portal vein branches is required for an effective PVE-associated hypertrophy of segments II and III. Here, we report a patient with an advanced IHC in the right liver lobe and segment IV and a partial portal vein thrombosis of liver 
segments II and III after selective PVE of liver segments IV-VIII resulting in an ischemic-type left lateral liver atrophy. However, extended right hepatectomy following PVE was made possible due to a massive hypertrophy of the caudate lobe.

\section{Case report}

A 65-year old female patient was referred to our center in April 2010 with a histopathologically confirmed, moderately differentiated cholangiocarcinoma of the right liver lobe and segment IV. The initial symptoms of the patient were reported as upper abdominal tension. The patient appeared to be in a good general condition and, except for a cholecystolithiasis, no further diseases were evident. The blood tests revealed elevated serum levels for carbohydrate antigen 19-9 (136 U/ml), normal findings for alpha-1fetoprotein and carcinoembryonic antigen, and slightly elevated liver enzymes with a normal bilirubin level (Table 1).

Computed tomography (CT) of the abdomen showed a tumor spreading over liver segments IV-VIII with a maximum diameter of $9 \mathrm{~cm}$ (Fig. 1a). Consecutive CT volumetry of the liver revealed a total liver volume (TLV) of $1,800 \mathrm{~cm}^{3}$ and an FLRV (liver segments I-III) of only $300 \mathrm{~cm}^{3}$. The tumor volume was $430 \mathrm{~cm}^{3}$. The TLV excluding tumor volume was $1,370 \mathrm{~cm}^{3}$. This made a ratio of FLRV per TLV excluding tumor volume of $21.9 \%$. In addition, a preoperatively performed biopsy of the liver revealed fibrotic tissue and intracytoplasmatic siderosis. The caudate lobe and left lateral segments II and III were free of tumor. The diagnostic evaluation revealed no extrahepatic tumor manifestation. In the liver hilus, the portal vein was in direct proximity to the tumor mass. The common hepatic artery and the extrahepatic portal vein showed no abnormalities. Furthermore, the portal vein branch to segment I diverted in direct proximity to the bifurcation of the main portal vein trunk (Fig. 1e). Due to insufficient FLRV an embolization of the portal branches to liver segments IV-VIII was performed (Fig. 1d, e). Because of an increased risk for an accidental embolization of the left-sided portal vein branches to segments II and III we did not perform initial embolization of segment I. Furthermore, an embolization of the caudate lobe would have additionally deprived the future liver remnant (FLR) from valuable healthy volume. We used non-calibrated particles to embolize the portal vein branches. Histoacryl coils or vascular plugs were also avoided because of the risk of accidental embolization of the left-sided liver segments, which might eventually compromise a successful hypertrophy and render the hepatic resection impossible.

Three weeks after embolization a CT volumetry of the liver displayed a FLRV of liver segments I-III of $380 \mathrm{~cm}^{3}$ and a TLV of $1,709 \mathrm{~cm}^{3}$. Interestingly, liver hypertrophy was evident only in the caudate lobe, but not in liver segments II and III where the CT scan revealed a constriction of the portal branches to liver segments II and III to be likely responsible for an inadequate left lateral hypertrophy. Prior to surgery it remained unclear whether the constriction of the left portal branches was due to tumor-related thrombosis or infiltration.

It was decided to perform an explorative laparotomy, where the tumor manifestation in liver segments IV-VIII was confirmed (Fig. 2a). No evidence for extrahepatic tumor dissemination or lymph node metastases was found. Further exploration confirmed a massive hypertrophy of the caudate lobe with a pre-existing atrophy of liver segments II and III (Fig. 2b). Thrombosis of the portal vein to segment II and III, as suspected in the CT scan, was confirmed by intraoperative ultrasound.

Preparation and exposition of the portal vein bifurcation was completed, followed by a thrombectomy of the left portal vein. A lymphadenectomy from the hepatic hilum to
Table 1 Liver function 1 day before and after PVE as well as 1 day before hepatic resection and on postoperative days 2 (POD 2) and 14 (POD 14)

ASAT aspartate amino transferase, $A L A T$ alanine amino transferase, $A P$ alkaline phosphatase, GGT gamma glutamyl transferase

\begin{tabular}{|c|c|c|c|c|c|c|}
\hline & \multirow[t]{2}{*}{ Normal values } & \multicolumn{2}{|l|}{ PVE } & \multicolumn{3}{|c|}{ Hepatic resection } \\
\hline & & 1 day before & 1 day after & 1 day before & POD 2 & POD 14 \\
\hline Bilirubin & $<17.1 \mu \mathrm{mol} / 1$ & 5.9 & 11.4 & 8 & 12.6 & 11.4 \\
\hline Total protein in serum & $66.0-87.0 \mathrm{~g} / 1$ & 69.9 & 72.5 & 75.2 & 60.1 & 59.3 \\
\hline Albumin in serum & $35-52 \mathrm{~g} / 1$ & 44.5 & 44.4 & 47.7 & 30 & 29.5 \\
\hline ASAT & $0.17-0.60 \mathrm{ukat} / \mathrm{l}$ & 0.71 & 1.28 & 0.63 & 5.08 & 0.53 \\
\hline ALAT & $0.17-0.60 \mathrm{ukat} / 1$ & 0.64 & 1.54 & 0.67 & 6.79 & 0.75 \\
\hline $\mathrm{AP}$ & $0.58-1.74$ ukat/l & 2.93 & 3.19 & 3.21 & 1.64 & 1.66 \\
\hline GGT & $<0.65$ ukat/l & 3.44 & 3.67 & 4.28 & 1.95 & 2.14 \\
\hline Lipase & $<1.0$ ukat/l & 0.63 & 0.56 & 0.97 & 1.88 & 1.26 \\
\hline Creatinine & $45-84 \mu \mathrm{mol} / 1$ & 50 & 48 & 56 & 43 & 91 \\
\hline Prothrombin time & $>70 \%$ & 118 & 122 & 113 & 69 & 91 \\
\hline
\end{tabular}



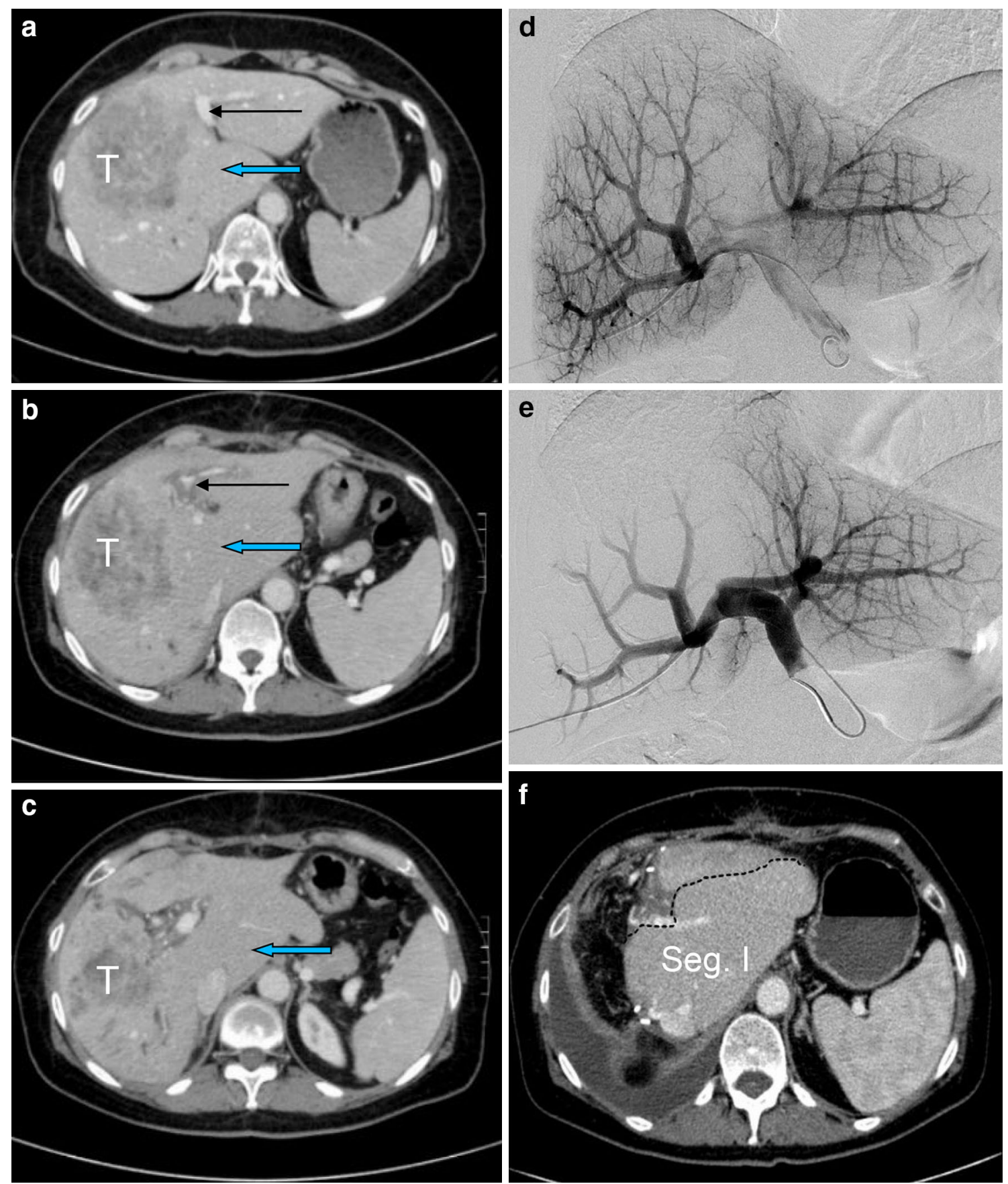

Fig. 1 a Computed tomography (CT) of the abdomen displaying an extended tumor manifestation prior to portal vein embolization. b CT of the abdomen 3 weeks after portal vein embolization. c CT of the abdomen 8 weeks after PVE and directly before extended right hepatectomy. d Antegrade transhepatic portography (with 4F pigtail catheter in the main portal vein trunk) showing normal contrasting of the patient's liver. e Antegrade transhepatic portography (with $4 \mathrm{~F}$ sidewinder catheter in the main portal vein trunk) showing no more

the celiac trunk was also performed. Even after portal thrombectomy, a very low blood flow was noted in the portal branches to liver segments II and III, likely due to a

contrasting of the right-sided liver parenchyma. f CT of the abdomen 3 months after extended right hepatectomy and curative resection. Black arrow tumor-free portal vein branches to segments II and III (a); a tumor-related partial thrombosis of the portal vein branches to segments II and III (b). Block arrow segment I prior to embolization (a), after extensive hypertrophy following embolization (b, c). Dashed line border between segment II/III and segment I. $T$ tumor, Seg I liver segment I

tumor-associated portal vein stenosis. The caudate lobe displayed a significant hypertrophy with powerful perfusion via both portal vein and proper hepatic artery. 
The operation was terminated as an exploratory laparotomy due to the ischemic-type atrophy of segments II and III associated with an expected marginal residual liver function of the FLR and an increased risk of PLF. The analysis of the intraoperative frozen sections and the definite histology of the thrombus and the excised lymph nodes showed no evidence of tumor manifestation. A control magnetic resonance imaging of the liver displayed a renewed closure of the portal vein branches to segments II and III.

Four weeks after the laparotomy and 7 weeks after PVE, another CT scan of the abdomen was performed for restaging and renewed volumetric analysis. Again the CT scan revealed a partial thrombosis of the portal branches to segments II and III (Fig. 1b); however, the tumor showed no progression with no tumor extension to segments II and
III or to the caudate lobe. The CT volumetry revealed a FLRV for liver segments I-III of $400 \mathrm{~cm}^{3}$ and a TLV of $1,250 \mathrm{~cm}^{3}$ (tumor volume excluded). This made a ratio of FLRV per TLV excluding tumor volume of $32 \%$. Hypertrophy of the FLR was solely due to an enlargement of segment I (Figs. 1c, 2c). Despite the ischemic-type atrophy of segments II and III we decided to perform an extended right hepatectomy with curative intent.

During the second operation, we explored the abdominal cavity and excluded extrahepatic tumor manifestations. As the intraoperative ultrasound confirmed the restriction of the tumor to segments IV-VIII, we affirmed the indication to perform an extended right hepatectomy. Parenchymal transection was performed using an ultrasonic dissection device (Cavitron Ultrasonic Surgical Aspirator, CUSA ${ }^{\circledR}$, Valleylab, Boulder, CO, USA) without any intraoperative
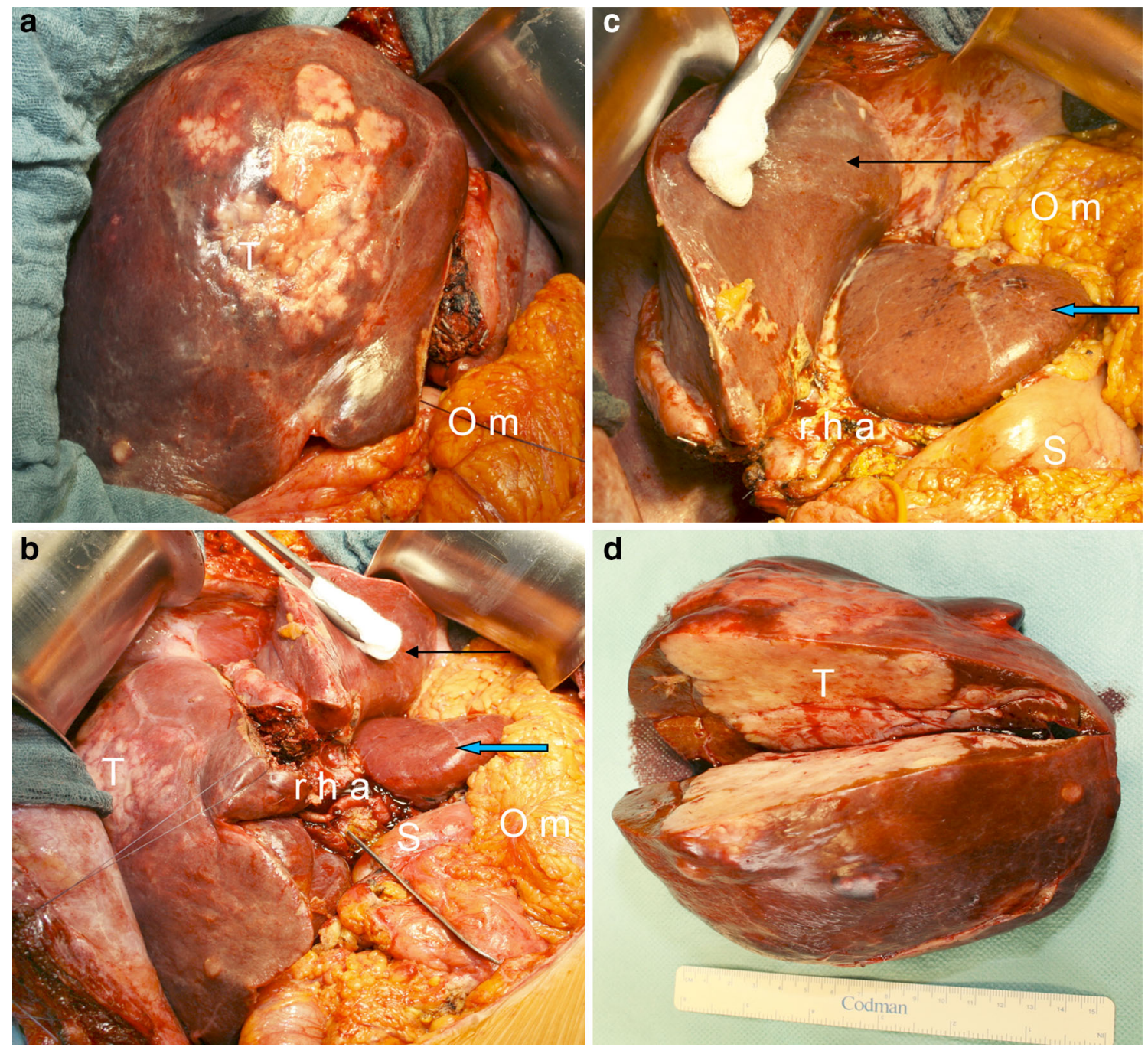

Fig. 2 a Tumor manifestation in the right liver lobe before resection. b Extensive hypertrophy of segment I, atrophic left lateral section (segment II and III) and the already isolated right hepatic artery prior to division. c Situs after hepatic resection with prominent caudate lobe, atrophic segments II and III and stump of the right hepatic artery after division. d Hepatectomy specimen after curative resection. $T$ tumor, $O \mathrm{~m}$ Omentum majus, block arrow segment I, black arrow segments II and III, $r h$ a right hepatic artery, $S$ stomach 
complications. Due to a risk of biliary leakage a $\mathrm{T}$ drain was routinely inserted in the main bile duct for decompression and easy access cholangiography.

In the postoperative course no signs of insufficient liver function were notable. During the postoperative stay in the intensive care unit the patient did not develop any significant complications. Ten days after resection, the patient developed a small abscess at the resection surface which was successfully treated by percutaneous drainage. There was no bilioma or bile leak evident. The postoperative duplex ultrasound of the liver revealed a powerful orthotopic flow of the left hepatic artery and of the hepatic veins and no evidence for central portal vein thrombosis but a persistent thrombosis in the left portal vein trunk. The conclusive pathological result showed a poorly differentiated cholangiocarcinoma without lymph node metastases (UICC stage II), hepatocellular steatosis and siderosis as well as cholestasis and cholangitis. The resection was categorized as formally curative (Figs. 1f; $2 \mathrm{c}, \mathrm{d})$. After a total postoperative hospitalization of 30 days the patient was dismissed in good general condition. The follow-up visits at 6,12 and 18 months after resection revealed normal liver function and no evidence of recurrent disease.

\section{Discussion}

Liver resection represents the only curative treatment option for IHC and is currently considered as the treatment of choice in this tumor entity. Studies about the efficacy of liver resection in patients with IHC showed long-term survival rates ranging from $30-60 \%$ at 5 years after resection [15-17]. Even in the presence of a more advanced tumor stage, partial hepatectomy can provide long-term survival in a considerable share of patients and the growing experience in liver surgery facilitates extended hepatectomies and provides a chance of cure in formerly non-resectable liver malignancies [15]. The published results of large series of patients who received liver resection within the last 2 years underlined not only the therapeutic efficacy of this treatment but also its safety. The mortality rate even after major liver resections is mostly $<5 \%$ in high-volume centers [18]. Hepatic surgery in the current era is regarded as a safe procedure, and its role in primary and secondary liver cancers is well established [19].

However, PLF remains a potentially life-threatening complication and often proves fatal, especially after major hepatectomy [18]. The risk of PLF depends on the quality of the parenchyma and the volume of the remnant liver. Once liver failure is established, no reliable disease- specific therapy is currently available and liver transplantation is mostly contraindicated in these patients, resulting in reported mortality rates of $60-80 \%[20,21]$. Thus, a sufficient FLRV is crucial for the success of major hepatectomies in these patients. Few treatment modalities are established to minimize the risk of postoperative liver insufficiency by improving liver function and the remnant volume before major hepatectomies, such as PVE and selective biliary decompression in patients with biliary stasis [22]. Recently, novel strategies have been proposed, i.e., inclusive CD133+ hematopoietic stem cells therapy, which seems to positively impact overall outcomes after extended right hepatectomy or in situ split techniques in combination with portal vein ligature [23-25].

Here, we propose a selective hypertrophy of the caudate lobe as a novel approach to facilitate extended right hepatectomy in patients with large tumors of the right liver lobe. Extended right hepatectomy may be considered impossible in cases of ischemic-type atrophy of left lateral segments. However, in contrast to liver segments II and III, the caudate lobe appeared well perfused and of good parenchymal quality in the present patient. Due to a significant hypertrophy of segment I during the post-interventional course following PVE, extended right hepatetcomy became feasible with no major complications apparent postoperatively. In particular, the liver function did not reveal any signs of significant insufficiency in spite of the atrophy of segments II and III.

This case is of major interest for patients with severely limited left lateral liver function scheduled for uni- or bilobular hepatectomies. These patients are widely excluded from curative treatment strategies due to the anticipated risk of PLF. Therefore, an induction of hypertrophy of segment I could be a promising approach and, unless the diagnosis is hilar cholangiocarcinoma, may provide a sufficient remnant liver function to perform curative liver resection. How can hypertrophy of segment I be induced tactically? Aware of the fact that isolated, massive hypertrophy of segment I following PVE is possible, initial embolization of this segment could be avoided. In selected patients, one could consider initial embolization of segments $4-8$ only, thus awaiting potential hypertrophy induction of segment I.

In conclusion, extended right hepatectomy in the presence of thrombosis of the left portal vein branches to segments II and III is feasible if liver segment I reveals an adequate hypertrophy. To our knowledge, this is the first case in which an extended right hepatectomy was made possible by massive PVE-associated hypertrophy of the caudate lobe. Therefore, atrophy of segments II and III may not represent a general contraindication to extended right hepatectomy. 
Disclosures Conflict of Interest: All authors (Georgi Atanasov, Moritz Schmelzle, Armin Thelen, Georg Wiltberger, Hans-Michael Hau, Felix Krenzien, Tim-Ole Petersen, Michael Moche, Sven Jonas) declare that they have no conflicts of interest.

Human/Animal Rights: This paper contains no data about human subjects that needs approval by appropriate ethics committee.

Informed Consent: The patient gave his Informed Consent prior to the study.

\section{References}

1. Halazun KJ, Al-Mukhtar A, Aldouri A, Malik HZ, Attia MS, Prasad KR, et al. Right hepatic trisectionectomy for hepatobiliary diseases: results and an appraisal of its current role. Ann Surg. 2007;246:1065-74.

2. Neuhaus P, Jonas S, Bechstein WO, Lohmann R, Radke C, Kling $\mathrm{N}$, et al. Extended resections for hilar cholangiocarcinoma. Ann Surg. 1999;230:808-19.

3. Hemming AW, Reed AI, Howard RJ, Fujitsa S, Hochwald SN, Caridi JG, et al. Preoperative portal vein embolization for extended hepatectomy. Ann Surg. 2003;237:686-93.

4. Wei AC, Poon RT, Fan ST, Wong J. Risk factors for perioperative morbidity and mortality after extended hepatectomy for hepatocellular carcinoma. Br J Surg. 2003;90:33-41.

5. Poultsides GA, Zhu AX, Choti MA, Pawlik TM. Intrahepatic Cholangiocarcinoma. Surg Clin North Am. 2010;90(4):817-37.

6. Kopek N, Holt MI, Hansen AT, Hoyer M. Stereotactic body radiotherapy for unresectable cholangiocarcinoma. Radiother Oncol. 2010;94(1):47-52.

7. Lang H, Sotiropoulos GC, Sgourakis G, Schmitz KJ, Paul A, Hilgard P, et al. Operations for intrahepatic cholangiocarcinoma: single-institution experience of 158 patients. J Am Coll Surg. 2009;208(2):218-28

8. Shen WF, Zhong W, Xu F, Kan T, Geng L, Xie F, et al. Clinicopathological and prognostic analysis of 429 patients with intrahepatic cholangiocarcinoma. World J Gastroenterol. 2009;15(47):5976-82.

9. Zhou XD, Tang ZY, Fan J, Zhou J, Wu ZQ, Qin LX, et al. Intrahepatic cholangiocarcinoma: report of 272 patients compared with 5,829 patients with hepatocellular carcinoma. J Cancer Res Clin Oncol. 2009;135:1073-80.

10. Yedibela S, Demir R, Zhang W, Meyer T, Hohenberger W, Schönleben F. Surgical treatment of massforming intrahepatic cholangiocarcinoma: an 11-year Western single-center experience in 107 patients. Ann Surg Oncol. 2009;16(2):404-12.

11. Jang JS, Lim HY, Hwang IG, Song HS, Yoo N, Yoon S. et al Gemcitabine and oxaliplatin in patients with unresectable biliary cancer including gall bladder: a Korean Cancer Study Group phase II trial. Cancer Chemother Pharmacol. 2010;65(4):641-7.

12. Kim JH, Yoon HK, Sung KB, Ko GY, Gwon DI, Shin JH, et al. Transcatheter arterial chemoembolization or chemoinfusion for unresectable intrahepatic cholangiocarcinoma: clinical efficacy and factors influencing outcomes. Cancer. 2008;113(7):1614-22.

13. Saxena A, Bester L, Chua TC, Chu FC, Morris DL. Yttrium-90 radiotherapy for unresectable intrahepatic cholangiocarcinoma: a preliminary assessment of this novel treatment option. Ann Surg Oncol. 2010;17(2):484-91.

14. Jonas S, Benckert C, Thelen A, Lopez-Hänninen E, Rösch T, Neuhaus P. Radical Surgery for hilar cholangiocarcinoma. Eur J Surg Oncol. 2008;34(3):263-71.

15. Jonas S, Thelen A, Benckert C, Biskup W, Neumann U, Rudolph B, et al. Extended Liver Resection for Intrahepatic Cholangiocarcinoma: A Comparison of the Prognostic Accuracy of the Fifth and Sixth Editions of the TNM Classification. Ann Surg. 2009;249:303-9.

16. DeOliveira ML, Cunningham SC, Cameron JL, Kamangar F, Winter JM, Lillemoe KD, et al. Cholangiocarcinoma: thirty-one year experience with 564 patients at a single institution. Ann Surg. 2007;245:755-62.

17. Nakeeb A, Pitt HA, Sohn TA, Coleman J, Abrams RA, Piantadosi $\mathrm{S}$, et al. Cholangiocarcinoma. A spectrum of intrahepatic, perihilar and distal tumors. Ann Surg. 1996;224:463-73.

18. Jarnagin WR, Gonen M, Fong Y, DeMatteo RP, Ben-Porat L, Little $\mathrm{S}$, et al. Improvement in perioperative outcome after hepatic resection: analysis of 1,803 consecutive cases over the past decade. Ann Surg. 2002;236:397-406.

19. Kavolius J, Fong Y, Blumgart LH. Surgical resection of metastatic liver tumors. Surg Oncol Clin N Am. 1996;5(2):337-52.

20. Otsuka Y, Duffy JP, Saab S, Farmer DG, Ghobrial RM, Hiatt JR, et al. Postresection hepatic failure: successful treatment with liver transplantation. Liver Transpl. 2007;13:672-9.

21. Balzan S, Belghiti J, Farges O, Ogata S, Sauvanet A, Delefosse $\mathrm{D}$, et al. The "50-50 criteria" on postoperative day 5: an accurate predictor of liver failure and death after hepatectomy. Ann Surg. 2005;242:824-8.

22. Schmelzle M, Duhme C, Junger W, Salhanick SD, Chen Y, Wu $\mathrm{Y}$, et al. CD39 modulates hematopoietic stem cell recruitment and promotes liver regeneration in mice and humans after partial hepatectomy. Ann Surg. 2013;257(4):693-701.

23. am Esch JS, Schmelzle M, Fürst G, Robson SC, Krieg A, Duhme $\mathrm{C}$, et al. Infusion of CD133+ bone marrow-derived stem cells after selective portal vein emolization enhances functional hepatic reserves after extended right hepatectomy: a retrospective single-center study. Ann Surg. 2012;255(1):79-85.

24. Schnitzbauer AA, Lang SA, Goessmann H, Nadalin S, Baumgart $\mathrm{J}$, Farkas SA, et al. Right portal vein ligation combined with in situ splitting induces rapid left lateral liver lobe hypertrophy enabling 2-staged extended right hepatic resection in small-forsize settings. Ann Surg. 2012;255(3):405-14.

25. Knoefel WT, Gabor I, Rehders A, Alexander A, Krausch M, Schulte am Esch J, et al. In situ liver transection with portal vein ligation for rapid growth of the future liver remnant in two-stage liver resection. Br J Surg. 2013;100(3):388-94. 